\title{
Experimental determination of the hydrofoil's angle of attack in the case of a turtle-like Autonomous Underwater Vehicle
}

\section{Conference Paper}

Author(s):

Font, D.; Tresanchez, M.; Siegenthaler, C.; Teixido, T.; Palleja, T.; Pradalier, Cédric; Palacin, J.

Publication date:

2011

Permanent link:

https://doi.org/10.3929/ethz-a-010025264

Rights / license:

In Copyright - Non-Commercial Use Permitted

Originally published in:

https://doi.org/10.1109/Oceans-Spain.2011.6003460 


\title{
Experimental determination of the hydrofoil's angle of attack in the case of a turtle-like Autonomous Underwater Vehicle
}

\author{
D. Font ${ }^{1}$, M. Tresanchez ${ }^{1}$, C. Siegentahler ${ }^{2}$, M. Teixidó ${ }^{1}$, T. Pallejà ${ }^{1}$ C. Pradalier ${ }^{2}$, J. Palacín $^{1}$ \\ ${ }^{1}$ Department of Computer Science and Industrial Engineering, University of Lleida, Jaume II, 69, 25001 Lleida, Spain \\ ${ }^{2}$ Autonomous System Lab. Tannenstrasse 3 CLA E 14. 8092 Zürich, Switzerland
}

\begin{abstract}
In this paper a turtle's hydrofoil 2 DoF's mechanism (degrees of freedom) is implemented and tested in a water channel as a propulsion system for an Autonomous Underwater Vehicle (AUV). The experiments carried out showed an optimal empirical value of the angle of attack for the turtle's hydrofoil that is compared with the theoretical value. The hydrofoil path used in the tests was a linear displacement and the optimization of this path will be analyzed in future works.
\end{abstract}

\section{INTRODUCTION}

The design and development of Autonomous Underwater Vehicles (AUV) has gained a lot of interest in the robotics' field and it can become an important tool for a wide range of applications such as: chemical water analysis, harbor surveillance, ship inspection, pipeline inspection, reef inspection, ship wreck research and archeology research. Depending on the autonomy, the AUV can even address large applications such as temperature mapping, topology mapping, pollution mapping, resource search missions, pollution search mission and general exploratory missions.

Most underwater vehicles use propellers like a propulsion system because they have high efficiency. However, they are an unsuited solution to carry out applications in underwater robots because they have to work under low flow conditions, in confined spaces, near the surface, and in unsteady flow [1]. The flapping foil is an alternative which is being studied in order to improve the locomotion propulsion system of AUV. It is focus on simulating the movement in a more stable way with less noise and good flexibility. Another advantage is the high maneuvering at the same time that is able to move with high efficiency [1].

\section{A. Biological background}

The AUV in study in this work pretends to have the appearance of a turtle with as many similarities as possible between the robot and the animal anatomic [2]. This project is focused on the hydrofoil (forelimb) part of a turtle's body which is the power source of the propulsion. It has some characteristics in their skeleton construction, for example the humerus and the radius are thicker and not too long [2].

Turtles move due to the thrust generated by the hydrofoils following a specific path depending on the specie of the turtle [3]. In the locomotion of freshwater turtle a shape as a circumference is followed for each leg (Fig. 1-a) compared to the marine turtle where a shape like an eight is reached (Fig. 1b), and both drag and lift forces are involved [4]. The path followed by the hydrofoils can be divided in four different phases [5] and are [6] called: downstroke, pronation, upstroke and supination. Downstroke and upstroke are the most important whereas pronation and supination are just transient movements to close the shape. Reference [4] and [5] studied the thrust force generated by the hydrofoil and the path modeled.

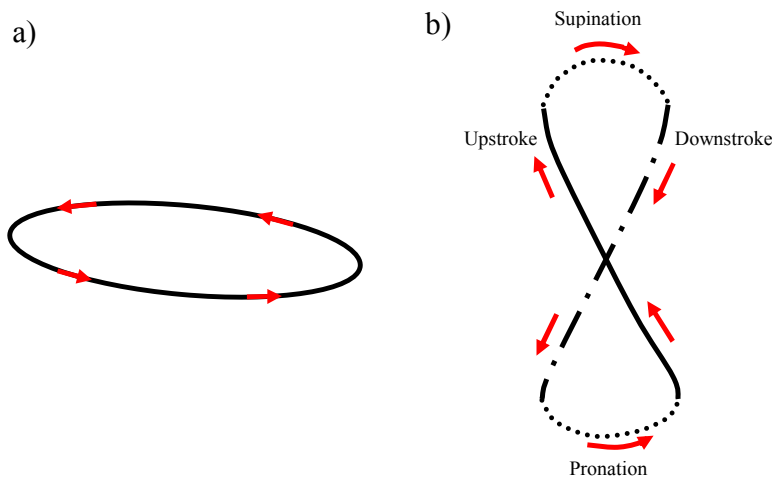

Figure 1. Hydrofoil's path for: a) freshwater turtle, b) sea turtle.

\section{B. Contribution of this work}

This project is part of a larger study under development at the Autonomous System Lab (ASL), department at Eidgenössische Technische Hochschule (ETH), Zürich University. The final goal is the development of an AUV using fin propulsion in similar fashion as sea-turtles.

This work proposes a compact 2-3 DoF (degrees of freedom) mechanism applied to the turtle's hydrofoil. The hydrodynamic profile selected for the hydrofoil's turtle is the NACA 0014 because it is symmetrical and it provides the best relation between lift and drag forces for a specific Reynolds number which was consider acceptable for the targets of the project. Reference [12] used the same fin for an AUV.

\section{Related work}

The research of AUV is focused on giving solutions in the areas of ecological studies, structure inspections and military service. Some prototype examples are the Slocum [7] and Ictineu [8], both of them generate trust due to propellers but having different aims. Slocum is a small gliding AUV with large operational range which harvests its propulsive energy from the heat flow between the vehicle engine and the thermal gradient of the temperate of the tropical ocean. Ictineu is an 
AUV prototype that has been developed in order to satisfy four different missions. The robot must be able to move from a launch/release point and submerging, to pass through a $3 \times 4$ meter validation gate, to locate a cross situated on the bottom of the pool and dropping a marker over it and to locate a midwater target and contracting it with the AUV.

Other AUV as AQUA [9] and Madeleine [10] are examples of robots which do not use propellers as propulsion. AQUA uses paddles whereas Madeleine uses webbed legs which are feeding with a mechanism of $2 \mathrm{DoF}$. Madeleine is a prototype developed by a team from the Autonomous Undersea Systems Institute, Lee, New Hampshire, USA. The motivations of this project was the prediction of the efficient pitching fin operation in the range $3-4 \mathrm{~W} / \mathrm{N}$ that is possible to reach by proper sizing of the motors, and to build a platform that allows testing of biological predictions about fin based locomotion. AQUA is an ambitious robot that swims or walks due to his legs. The vehicle uses a variety of sensors to provide a range of real tasks in applications that require large autonomy.

\section{MATERIALS AND METHODS}

The materials and methods used in this study to implement and validated the propose hydrofoil are described. During the design phase different $\mathrm{CAD}$ programs were used such as JavaFoil, Working Modelm, Unigraphics NX 6, AutoCAD 2010, Ansys Workbench 11, Maple and MATLAB. To control the hydrofoil the motors used in this proposal were the Maxon EC $2250 \mathrm{~W} / 167129$ used in combination with a planetary gearhead GP 32C 190:1 and an optical encoder (MR Encoder 128 CPT) with two channels of 128 counts per revolution and one reference channel. The control of the positions of the motors was performed with three EPOS 24/5 from Maxon, with the hydrofoil's trajectory defined by custom software written in Matlab and $\mathrm{C}++$. Experiments were carried out in a water channel belongings to the ETH Zürich University.

\section{DESIGN AND IMPLEMENTATION OF THE TURTLE'S HYDROFOIL}

This section discusses which hydrodynamic profile is chosen as a turtle's hydrofoil based on the hydrodynamic theorems.

\section{A. Hydrodynamic profile and theoretical angle of attack}

The hydrodynamic profile of a turtle's hydrofoil in movement into the water defines the drag $\mathrm{D}$ and lift $\mathrm{L}$ forces (Fig. 2), and a resultant moment M. The lift is the force perpendicular to the flow direction that directly opposes the weight of the fin and acts through the center of pressure of the object. Along the flow direction and opposed to the thrust the drag force appeared. Drag acts in a direction that is opposite to the motion of the fin. The resultant moment is defined negative in counterclockwise. All these forces are applied at one point called pivoting point which is located at $25 \%$ of the chord. Equation (1) defines the relationship between the properties of the hydrodynamic profile and the characteristics of the water.

$$
\begin{aligned}
& L=\frac{1}{2} \cdot V^{2} \cdot \rho \cdot C_{l} \cdot S \\
& D=\frac{1}{2} \cdot V^{2} \cdot \rho \cdot C_{d} \cdot S \\
& M=\frac{1}{2} \cdot V^{2} \cdot \rho \cdot C_{m} \cdot S \cdot C
\end{aligned}
$$

where $\mathrm{V}$ is the speed of the object relative to the fluid; $\rho$ is the density of the fluid; $\mathrm{C}$ is the chord; $\mathrm{C}_{\mathrm{l}}, \mathrm{C}_{\mathrm{d}}$ and $\mathrm{C}_{\mathrm{m}}$ are the lift, drag and momentum coefficients respectively, and $\mathrm{S}$ is the surface of the fin. This surface is calculated by the chord for the length of the fin because the theory of thin profiles can be applied.

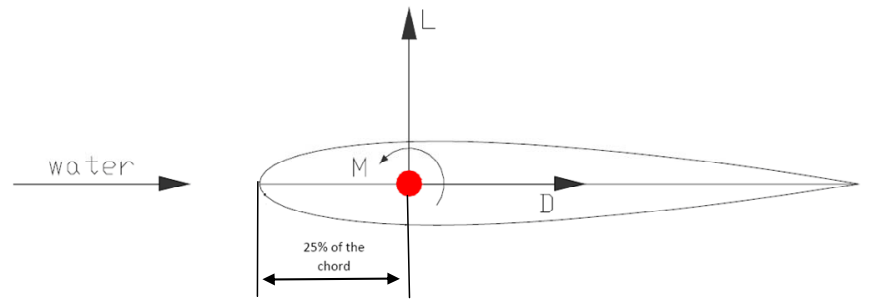

Figure 2. Hydrodynamic forces applied at the pivoting point of a NACA 0014 profile.

The hydrodynamic profile of the turtle's hydrofoil was the NACA 0014 because it is symmetrical and it provides the best relation between lift and drag forces. The hydrofoil's angle of attack $\alpha$ is defined when the lift divided by the drag coefficient is maximal $(\mathrm{L} / \mathrm{D})$ and due to JavaFoil is determined as $8^{\circ}$. More parameters are known (see Table I) depending on the Reynolds' number (2) and the hydrodynamic profile.

$$
\operatorname{Re}=\frac{\rho \cdot V_{w} \cdot L}{\mu}
$$

where $\rho$ is the density of the fluid; $V_{w}$ is the fluid velocity; $L$ is characteristic linear dimension of the profile and $\mu$ is the dynamic viscosity of the fluid.

Fig. 3 illustrates the evolution between the lift and drag coefficient $\left(\mathrm{C}_{1}\right.$ and $\left.\mathrm{C}_{\mathrm{d}}\right)$ changing the angle of attack between 0 to $10^{\circ}$. For a drag coefficient of 0.025 the lift coefficient grows until a value of 1 and then increases slowly until $C_{d}$ reach a value of 0.038 .

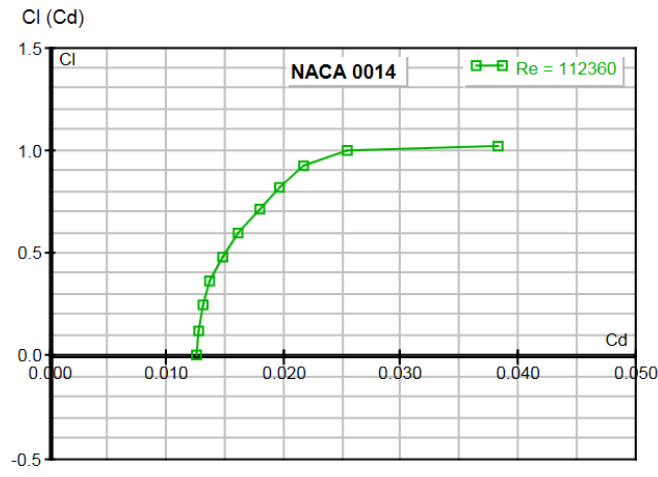

Figure 3. Evolution between $C_{1}$ and $C_{d}$ depending on the angle of attack. 
TABLE I

CHARACTERISTICS OF A NACA 0014 PROFILE FOR AN ANGLE OF ATTACK OF $8^{\circ}$

\begin{tabular}{|c|c|c|c|c|c|c|c|c|c|c|}
\hline $\boldsymbol{\alpha}$ & $\mathbf{C}_{\mathbf{l}}$ & $\mathbf{C}_{\mathbf{d}}$ & $\mathbf{C}_{\mathbf{m}}$ & $\mathbf{T . U}$ & $\mathbf{T . L}$ & $\mathbf{S . U}$ & $\mathbf{S . L}$ & $\mathbf{L} / \mathbf{D}$ & $\mathbf{A . C}$ & $\mathbf{C . P}$ \\
\hline 8 & 0.918 & 0.02 & -0.01 & 0.03 & 0.884 & 0.883 & 0.977 & 42.033 & 0.264 & 0.263 \\
\hline
\end{tabular}

\section{B. Turtle's hydrofoil path}

The path followed by the hydrofoil of a sea turtle has an eight shape and this profile was selected for the computation of the hydrodynamic forces applied. Fig. 4 shows a representation of the trajectory of the turtle's hydrofoil with three critical positions labeled. In all the cases the profile is titled following the description of the angle of attack. These situations are the most unfavorable since is when the speed of the profile relative to the water is higher and, the forces are calculated on the tip of the fin taking into account the hydrodynamic equations (1). The lift force, drag force and momentum for case 1 are $7.09 \mathrm{~N}$, $0.1687 \mathrm{~N}$ and $-0.0093 \mathrm{Nm}$ respectively. For case 2 are 6.8782 $\mathrm{N}, 0.1636 \mathrm{~N}$ and $-0.009 \mathrm{Nm}$ and, for case 3 they are $22.449 \mathrm{~N}$, $0.5341 \mathrm{~N}$ and $-0.0293 \mathrm{~N}$.

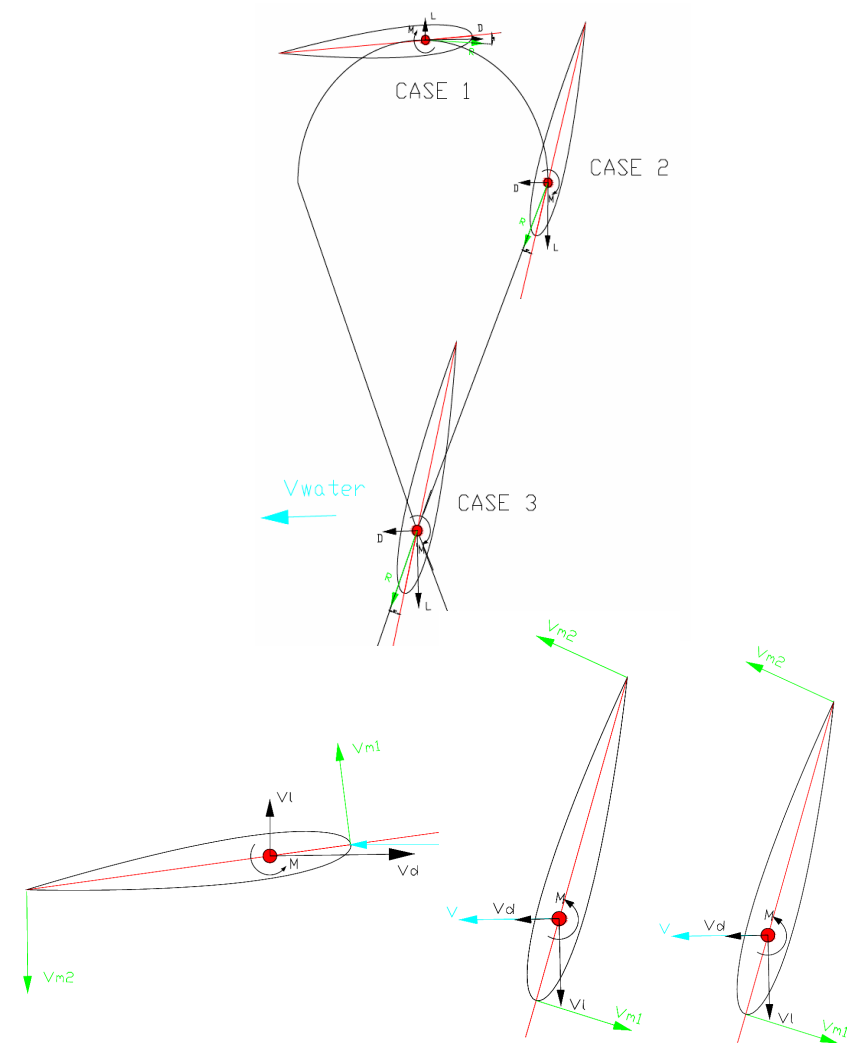

Figure 4. Body diagram of the hydrodynamic profile in the most unfavorable situations through the eight paths.

\section{HYDROFOIL MOTION MECHANISM}

This section analyzes the final mechanism implemented after being studied several alternatives. Terms as sealing, compactness and torque requirements are the most important concepts that are been compared. In each alternative different calculations and simulations have been done to estimate the torque value required by the motors and a decision matrix was created to select the final implementation.

\section{A. Final implementation}

Fig. 5 shows the final implementation of the complete turtle's hydrofoil and the mechanism. The motors used in this implementation were the Maxon EC $2250 \mathrm{~W}$. Each engine gives a different vertical, horizontal and rotational movement which is transmitted to the hydrofoil. The design of the whole mechanism depends on the maximum amplitude of the final motion, which, in this initial stage, is $60^{\circ}$ in both directions (vertical and horizontal). a)

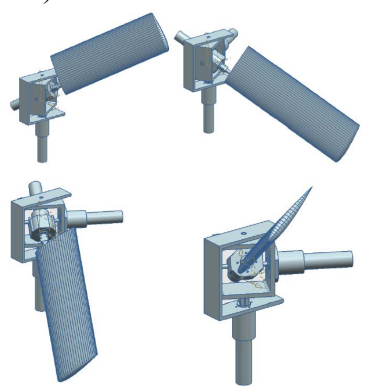

b)

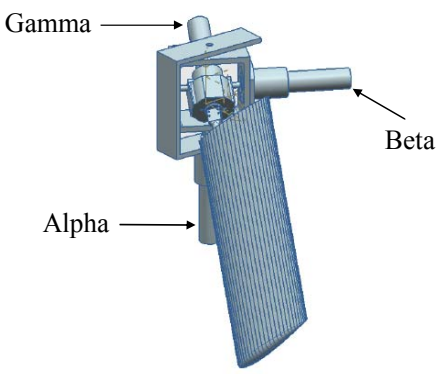

Figure 5. Extreme configurations of the mechanism implemented (a). Definition of the motor's name (b)

To ensure that each component have enough endurance to withstand the efforts, the von Misses stress results of the simulations with Ansys (Fig. 6) have to be higher than the yield stress of the material. That is why aluminum and steel were used in different parts. In order to realize the static mechanical analysis some considerations have been done. A solid rigid was created when there were not relative motions between the parts involved, so the study was carried out applying the forces as a unique element. The forces introduced correspond to the most unfavorable case which is when the hydrodynamic forces are applied on the tip of the fin. These forces were calculated following case number 3 . The weight of each element was applied to their center of gravity; these values were provided by NX 6 program. Before introducing the forces a fine mesh was created. a)

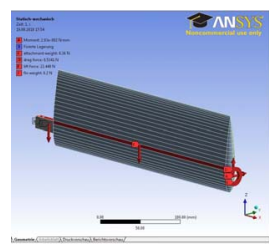

b)

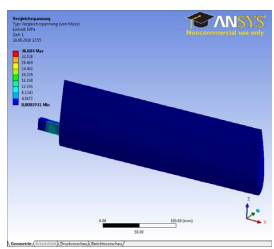

c)

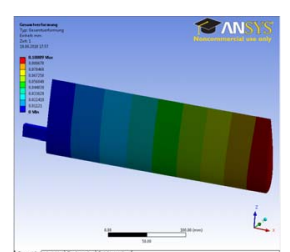

Figure 6. Static mechanical (a), equivalent stress (b) and deformational analysis (c) of a part of the mechanism. 


\section{EXPERIMENTAL}

In this phase different experiments have been carried out to empirically estimate the optimal value of the angle of attack.

\section{A. Approximation of the path's hydrofoil}

Fig. 7 shows the path's definition, it is a $2 \mathrm{DoF}$ approximation for the 8 turtle's motion. Fig. 8 shows the dynamic evolution of the angular position of the motors used to control the hydrofoil.
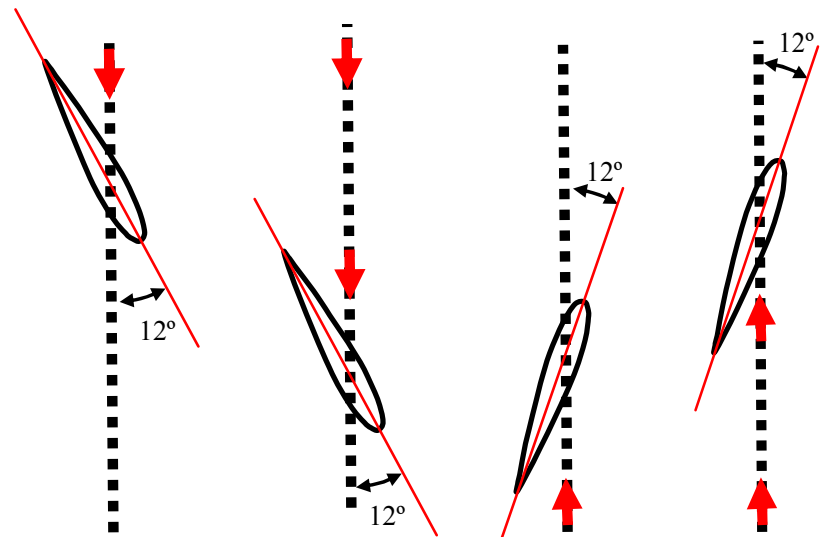

Figure 7. Path definition.

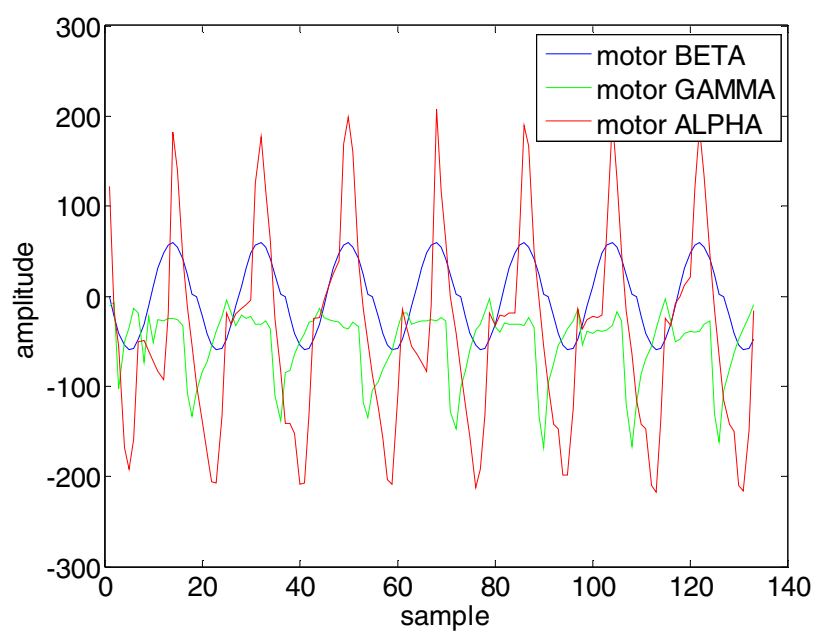

Figure 8. Angular position of the motors.

\section{$B$. Values reached during the experiments performance}

Fixing the mechanism in the water channel through a rigid structure keeping the turtle's hydrofoil submerged into the water as much as possible to get the best results and keeping the motors safe. Once the mechanism was fixed, the motors had free movement which was activated or deactivated depending on the path's hydrofoil was wanted to generate. Running the motion the current values of each motor (fig. 5) were extracted. Fig. 9, 10, and 11 show the current measured in each motor.
The expression of (3) was used to compute the mean current value of each motor: $57.7 \mathrm{~mA}, 67.9 \mathrm{~mA}$, and $183.3 \mathrm{~mA}$ for the gamma, alpha and beta motors.

$$
I_{\text {mean }}=\frac{\sum_{i=1}^{3} \frac{\sum_{j=1}^{N}\left|I_{i}(j)\right|}{N}}{3}
$$

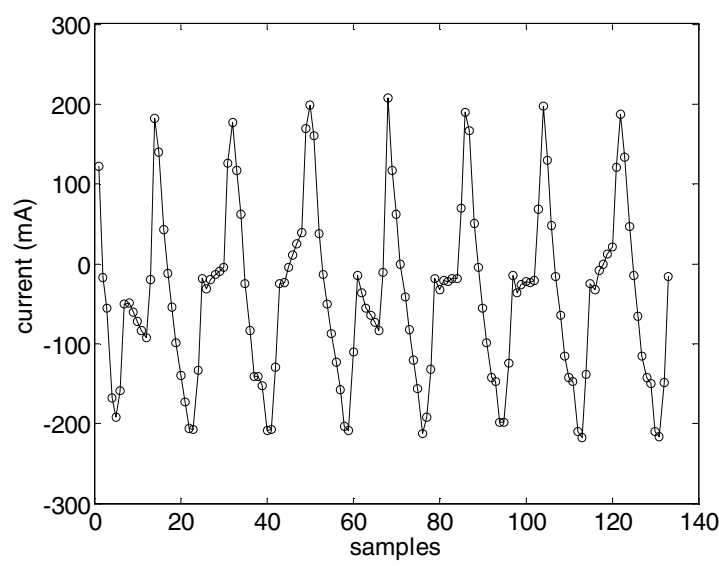

Figure 9. Current measured in the Gamma motor.

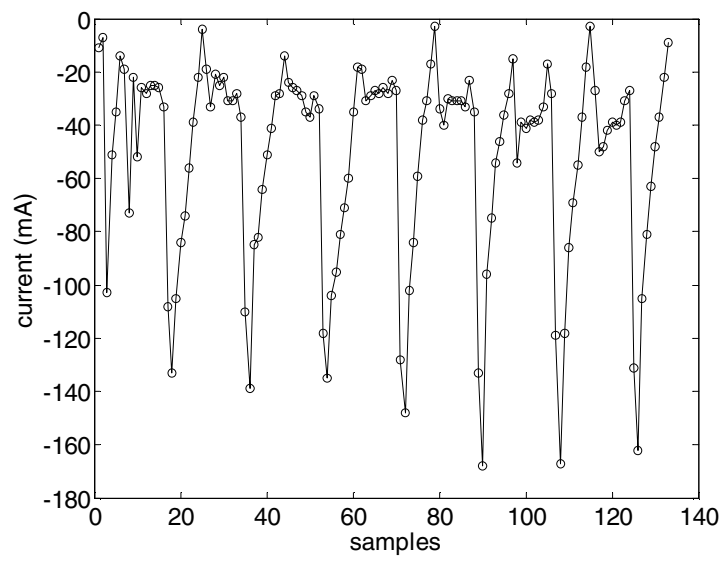

Figure 10. Current measured in the Alpha motor.

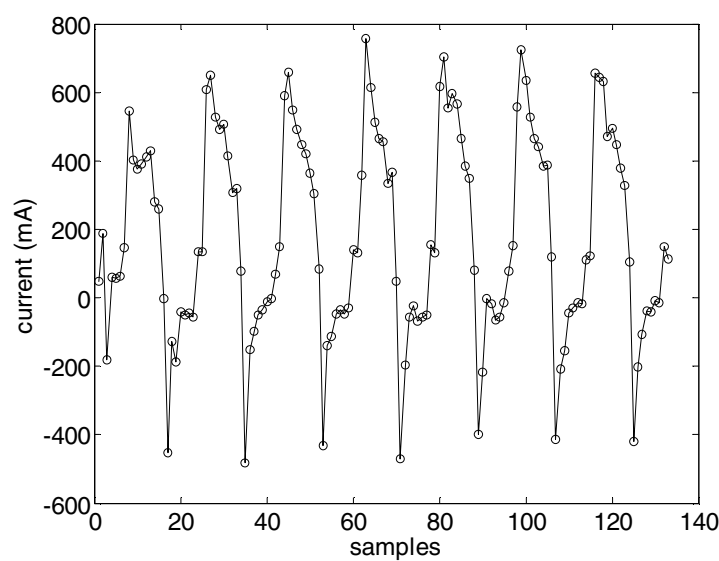

Figure 11. Current measured in the Beta motor. 
During the motion, the mechanism was generating thrust force along $\mathrm{X}$ axis. What it means the motor beta, which was not generating movement, was counteracting this force in both direction as its consumption show. It is supposed that the turtle's robot was moving along $\mathrm{X}$ axis, sometimes forwards and sometimes backwards, being the advance of the robot not optimal.

\section{Experimental validation of the optimal angle of attack}

The second experiment is focus on validating the theoretical optimal angle of attack. The same trajectory (Fig. 7) was run changing the angle of attack in $2^{\circ}$ each time from 2 up to $20^{\circ}$. Then, recollecting the current data from the EPOS and taking into account the consumption of the motor which counteracted the advance of the mechanism is decided which the optimal angle of attack is. This motor was counteracting the horizontal force composed by the horizontal component of the hydrodynamic forces (lift and drag), consequently it was counteracting thrust force which is the one which generates the motion on the robot. For this reason, when the current of this motor is higher and it is incrementing means that this is the optimal angle of attack since is when the mechanism is producing more force in one direction. The current results achieved in the water channel are the ones can be seen in Fig. 12. It can be inferred that the optimal angle of attack is $12^{\circ}$ because is where exist a peak indicating the maximum reached.

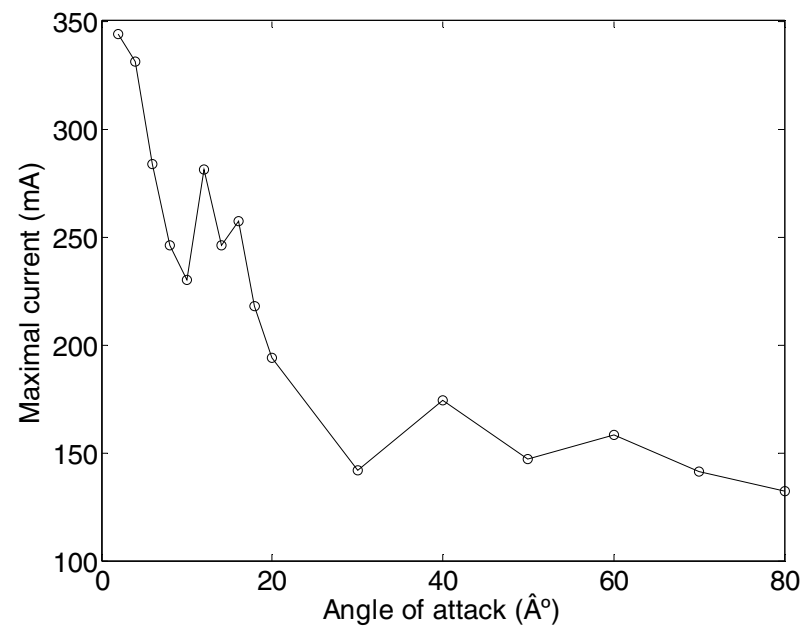

Figure 12. Maximal current of the beta motor relative to the angle of attack.

There is a difference of $4^{\circ}$ between the theoretical and empirical angle of attack. That is way some assumptions have been done during the calculations like the robot speed is constant with $1 \mathrm{~m} / \mathrm{s}$. In addition there are some limitations executing the JavaFoil program because it does not work with accurate results when you have model laminar separation bubbles and flow separation. Another point is that the experiments were perform in a water channel, where it is extracted an approximated information about the effects that the motion of the assembly should have in a open environment such as a lake or in the sea.

\section{CONCLUSIONS AND FUTURE WORK}

The use of the NACA 0014 foil as a turtle's hydrofoil and the 2-3DoF's mechanism were validated in different experimental tests. In this work, both theoretical and empirical values of the optimal angle of attack were tested and compared. The differences between both values are $4^{\circ}$ as a consequence of the assumptions and limitations applied in the theoretical evaluation performed.

The hydrofoil path used in the tests was a linear displacement; future work will study different alternatives involving the 3DoF's mechanism to optimized turtle's motion.

\section{REFERENCES}

[1] Stephen Licht, Victor Polidoro, Melissa Flores, Franz S. Hover, and Michael S. Triantafyllou, "Design and projected performance of a flapping foil AUV," IEEE Journal of oceanic engineering, vol. 29, pp. 3, July 2004.

[2] Jeanette Wyneken, "The anatomy of sea turtles," US. Department of Commerce National Oceanic and Atmospheric Administration, National Marine Fisheries Service, December 2001.

[3] J. Davenport, Sarah A. Munks and P. J. Oxford, "A comparison of the swimming of marine and freshwater turtles," Proc. R. Soc. London, vol. B 220, pp. 447-475, 1984.

[4] Dinghui Chu, Xiaobai Liu and Mingjun Zhang, "Research on turtle hydrofoil motion principle and bionics," Proceedings of the IEEE International Conference on Automation and Logistics, China, August 18 $-21,2007$.

[5] Mingjun Zhang, Xiaobai Liu, Dinghui Chu and Shaobo Guo, "The principle of turtle motion and bio-mechanism of its four limbs research," College of Mechanical and Electrical Engineering, Harbin Engineering University, 2008.

[6] Jian'an Xu, Xiaobai Liu, Dinghui Chu, Lining Sun and Mingjun Zhang, "Analysis and experiment research of the turtle forelimb's hydrofoil propulsion method," Proceedings of the 2009 IEEE International Conference on Robotics and Biomimetics, China, December 19-23, 2009.

[7] Douglas C. Webb, Paul J. Simonetti and Clayton P. Jones, "SLOCUM: An underwater glider propelled by environmental energy," IEEE Journal of oceanic engineering, vol. 26, pp.4, October 2001.

[8] D. Ribas, N.Palomeras, P. Ridao, M. Carreras and E. Hernandez, "ICTINEUAUV wins the first SAUC- E competition," 2007 IEEE International Conference on Robotics and Automation, Italy, 10-14. April 2007.

[9] G. Dudek, P. Giguere, Ch. Prahacs et al., "AQUA: An amphibian autonomous underwater robots," Published by IEEE Computer Society, pp. 46-53, January 2007.

[10] Mathieu Kemp, Brett Hobson and John H. Long, "MADELEINE: An agile AUV propelled by flexible fins," Proceedings of the 14th International Symposium on Unmanned Untethered Submersible Technology (UUST). Autonomous Undersea Systems Institute, Lee, New Hampshire, USA. 2005.

[11] http://www.mh-aerotools.de/airfoils/javafoil.htm, Last access on 10 November 2010.

[12] Ryan Moody, "The design, construction, and testing of a flexible fin propelled autonomous underwater vehicle," North Carolina State University Integrated Manufacturing Systems Engineering Institute Raleigh NC, 27607. 